https://doi.org/10.32735/S0718-220120180004700172

161-176

\title{
LA ENFERMEDAD COMO RASGO HUMANO. HACIA UNA CONSIDERACIÓN DE LA ENFERMEDAD EN CUANTO FENÓMENO EXISTENCIAL
}

Sickness as a human trait. Towards a consideration of disease as existential phenomena

DIANA AURENQUE STEPHAN

Universidad de Santiago de Chile diana.aurenque@usach.cl

FRANÇOIS JARAN DUQUETTE

Universitat de Valencia (España)

francoisjaran@gmail.com

\section{Resumen}

En el presente trabajo se investigan los alcances y las consecuencias que se desprenden de utilizar a Heidegger en el campo de la filosofía de la medicina. Con este fin, la investigación se divide en los siguientes puntos: 1) se explicitará en qué puede consistir una "antropologización" del pensamiento heideggeriano; 2) se ofrecerá una descripción general respecto de las teorías de la salud y enfermedad más significativas y actuales en el campo de la teoría de la medicina; 3) nos detendremos en el análisis relacionado con el problema de la enfermedad desde una perspectiva heideggeriana, para finalmente, 4) ofrecer algunas reflexiones críticas en relación con el análisis ofrecido.

Palabras clave: Salud y enfermedad; Heidegger; fenómeno existencial; analítica del Dasein; humano y animal.

\section{Abstract}

In this contribution we investigate the scope and consequences that arise from applying Heidegger in the field of philosophy of medicine. For this purpose, the paper is divided in the following points: First, we will make explicit the meaning of an "anthropologization" of Heidegger's thought. Second, an overview of the most significant contemporary theories of health and disease in the field of theory of medicine will be offered. Third, we will interpret the meaning of disease from a Heideggerian perspective. Finally, 4) we will offer some critical reflections offered in relation to the analysis.

Key words: Health and disease; Heidegger; existential phenomenon; analytic of Dasein; human and animal.

\section{INTRODUCCIÓN: TEMA Y PROPÓSITO DEL TRABAJO}

Desde comienzos de los años 1990 el cuidado de la salud se ha vuelto un eje cada vez más importante en el plano de políticas de salud pública en varios países occidentales. Ahora bien, pese a que el vasto interés por mantener e incentivar una 
vida saludable parece constatar implícitamente que la salud es concebida como un valor claro y definido y, en consecuencia, como algo que debe ser cuidado, paradójicamente la díada salud-enfermedad se ha vuelto cada vez más difícil de comprender. Sobre todo en tiempos donde la medicina se apoya progresivamente en recursos técnicos para detectar enfermedades antes de su aparición sintomática o clínica (por ejemplo, por medio de diagnósticos predictivos genéticos), los conceptos de enfermedad y salud son más difíciles de acotar. A raíz de lo anterior, la presente investigación se propone reflexionar acerca del significado de salud y enfermedad en sentido filosófico, considerando por una parte las teorías de la salud más influyentes en la discusión bioética contemporánea, mas al mismo tiempo intentado enriquecer aquella discusión mediante la inclusión de una perspectiva hermenéuticafenomenológica.

Más concretamente, en el presente trabajo ofrecemos algo que se inscribe dentro de lo que, de forma general, podemos considerar como una reinterpretación antropológica de la filosofía heideggeriana y que persigue dos propósitos fundamentales: Por un lado intentamos investigar los alcances así como las posibles consecuencias que se desprenden de utilizar a Heidegger en el campo de la filosofia de la medicina, para así, por otro lado, enriquecer debates en torno al significado de salud y enfermedad en dicho campo. Proponemos concretamente investigar y elaborar lo que Heidegger llamó en Ser y tiempo el tratamiento de la enfermedad como "fenómeno existencial" (Heidegger 1927, p. 247) y que dejó sin desarrollar. En dicha obra, si bien Heidegger insiste en el fenómeno de la muerte y de la inconmensurabilidad de la relación que mantienen el animal y la existencia humana con ella, deja apartado sin embargo el tema de la enfermedad y su tratamiento como fenómeno existencial. La presente contribución se hace cargo de dicha tarea e intenta dilucidar qué significa y hasta qué punto la enfermedad puede constituir un "fenómeno existencial" y, por lo tanto, una experiencia ligada de forma estructural al Dasein.

Para ello, el trabajo se divide en los siguientes puntos: En un primer momento se explicitará brevemente en qué puede consistir la mencionada "antropologización" del pensamiento heideggeriano. En un segundo punto se ofrecerá una descripción general concerniente a las teorías de la salud y enfermedad más significativas y actuales en el campo de la teoría de la medicina. En un tercer momento nos detendremos en el análisis relacionado con el problema de la enfermedad desde una perspectiva heideggeriana y, finalmente, ofreceremos algunas reflexiones críticas en relación con el análisis ofrecido.

\section{2. ¿DASEIN ENFERMO? ANTROPOLOGÍA Y ANALÍTICA DEL DASEIN}

El planteamiento de nuestro estudio nos obliga a justificar en un primer momento el uso que hacemos de la filosofia heideggeriana - en concreto, del proyecto de una analítica del Dasein - en el campo de la filosofía de la medicina. Sabemos que el pensamiento del primer Heidegger está en gran parte dedicado a una dilucidación 
de la existencia humana y de la comprensión que tiene del ser, pero subraya de forma insistente la distancia que separa su analítica de la existencia humana a cualquier intento de abordar al ser humano desde una perspectiva antropológica, psicológica 0 biológica. A primera vista, el intento de utilizar su pensamiento para pensar los fenómenos de enfermedad y salud puede parecer arriesgado.

Si nos referimos a una de las caracterizaciones más completas que Heidegger realizó de su concepto de Dasein, la del curso de verano de 1928 (Heidegger, 1978), vemos explicado claramente ahí que si Heidegger emplea el término "existencia" (Dasein) en vez de "ser humano" (Mensch), es porque el objeto que ha de ser analizado debe permanecer "neutro". Esta "neutralidad" significa fundamentalmente que el Dasein "alberga la intrínseca posibilidad" de extenderse fácticamente dentro del espacio, dentro de un cuerpo y así tener género, pero que el estudio que propone Heidegger se sitúa más allá, es decir, en el Noch-nicht de dicho extenderse. Suponiendo, pues, que el Dasein es un ente que se sitúa en el "todavía no" de la espacialidad, de la corporalidad y de la sexualidad (Räumlichkeit, Leiblichkeit, Geschlechtlichkeit (Heidegger, 1978, p. 173), dificilmente podría su estudio decirnos cosas acerca de la enfermedad y de la salud. Sin embargo, parece que la enfermedad sea también un fenómeno de importancia al nivel ya no biológico, sino ontológico. En efecto, Heidegger deja entrever la posibilidad del estudio de la enfermedad como "fenómeno existencial".

$\mathrm{Si}$, en general, consideramos posible y productivo utilizar el pensamiento heideggeriano en el ámbito de la filosofía de la medicina, es decir, un contexto de corte "antropológico", es porque nos parece que Heidegger mismo nos invita a tal uso. De hecho, la "antropologización" del pensamiento heideggeriano es una tendencia que va ganando terreno en la literatura actual, a la vez que se va redescubriendo el planteamiento de la Antropología Filosófica, movimiento filosófico de principios del siglo XX liderados por Max Scheler y Helmuth Plessner (Fischer, 2009, p. 9).

Uno de los varios reproches que hace Heidegger a la tradición filosófica occidental es haber entendido la existencia humana a partir de su animalidad. Este sesgo filosófico, según Heidegger, no tendría su origen en la filosofía griega sino en el traslado de la filosofía griega al latín, es decir, su "reinterpretación metafísica" (Heidegger, 1976, p. 322). En efecto, Aristóteles hablaba como sabemos del ser vivo dotado de $\lambda$ ó ${ }^{\circ} \varsigma$-que, para Heidegger, es fundamentalmente lenguaje-, mientras que la filosofía romana hablará ya no de un ser vivo sino del animal rationale, es decir, un animal dotado no de lenguaje sino de ratio, de razón. Desde entonces, el ser humano no hubiera podido dejarse pensar desde su propia esencia, sino únicamente en una comparación con el animal. Esta tesis polémica la defiende a lo largo de toda su obra, presentando al "animal metafísico" de Schopenhauer o al "animal no establecido" de Nietzsche como dos de los últimos avatares de esta larga tradición.

Llamamos polémica esta tesis en la medida en que en la misma época en la que Heidegger la defiende en Ser y tiempo, otro movimiento filosófico también 
heredero en parte de la fenomenología husserliana defiende la tesis diametralmente opuesta. En efecto, la Antropología Filosófica considera que el error de la tradición filosófica ha sido justamente pensar al ser humano a partir del $\lambda$ ó ${ }^{\circ} \varsigma$-un rasgo que comparte con los dioses- en vez de hacerlo desde su animalidad ( $\varphi v ́ \sigma ı)$ ). La Antropología Filosófica pretende en este sentido romper con esta manera que tuvo la "burguesía griega" de pensar el ser humano como homo sapiens, es decir, como un ser cuyo rasgo fundamental sería racional y de origen divino (Scheler, 1926, p. 125-129; Gehlen, 1983). Según Scheler, esta teoría hubiera alejado el ser humano del animal, impidiendo que ponga de manifiesto lo que precisamente distingue el uno del otro.

Pero la teoría del Homo sapiens -en el sentido que aquí lo entiende Scheler-se tambalea a lo largo del siglo XIX. Scheler destaca dos momentos esenciales: primero, Hegel hubiera mostrado la historicidad de la razón humana, negándole el carácter inmutable y eterno que se asoció hasta Kant al concepto de razón. La razón no es siempre idéntica sino que se va construyendo a lo largo de la historia y necesita tiempo para llegar a ser sí misma. Segundo, la teoría de la evolución de Darwin permite, aunque sea de forma limitada, imaginar un desarrollo de la vida que caminaría desde los organismos más sencillos hasta la inteligencia humana. Scheler combate esta posibilidad, pero subraya, apoyándose en Dilthey, que la naturaleza divina de la razón no puede aparecer ya como una evidencia.

En los textos que redactó como preparación a su inacabada Antropología Filosófica (Scheler, 1928; Scheler, 1987), Scheler busca una forma de resaltar la singularidad y la dignidad de la especie humana frente al resto de los animales sin que este rasgo pueda reducirse a una simple evolución de la vida. El concepto de espíritu (Geist) que Scheler considera como propio del ser humano y que coloca en el fundamento de su antropología constituye precisamente esta facultad que distingue al ser humano del resto de la vida y que no se puede entender como el resultado de la última evolución de la vida. El espíritu opone más bien el ser humano al resto de los seres vivientes.

El debate que mantuvo Heidegger con la antropología filosófica está bien documentado. Pero es importante entender qué reprocha Heidegger al planteamiento de Scheler. De alguna forma, Heidegger también busca caracterizar al Dasein a partir de un rasgo que lo aleja del resto de los seres vivientes: la comprensión del ser. Aquí como en Scheler, el rasgo definitorio de la existencia humana no puede explicarse a partir de la teoría de la evolución de las especies y tampoco se piensa como una parcela divina, inmutable y eterna en el hombre, inmune a los cambios históricos.

El meollo del debate con la Antropología Filosófica se debe buscar en otro sitio. Una lectura de la cuarta y última parte de la obra Kant y el problema de la metafisica permite entender que aunque Scheler y sus colaboradores hayan ofrecido una antropología interesante y destacado de forma brillante la problematicidad del ser humano (Heidegger inserta un gran número de referencias a Scheler en el $\S 37$ de la 
obra), el calificativo de "filosófica" es, sin embargo, usurpado. En efecto, según Heidegger, si una antropología ha de ser filosófica, tendrá que emprender inicialmente una determinación de la esencia de la filosofía que le pueda dar pistas acerca de cómo se debe interrogar la existencia humana dentro de un marco filosófico. La antropología "filosófica" que propone Scheler dice cosas interesantes acerca del ser humano pero no sabe lo que está buscando, cuál es su meta verdadera, ya que no procuró nunca legitimar sus pretensiones filosóficas. ¿En qué sentido es filosófica esta antropología? Heidegger defenderá que si fuese realmente filosófica, debería estar preocupándose por la pregunta acerca del ser y, en consecuencia, de la relación que mantiene el ser humano con esta pregunta.

Vemos que la divergencia entre el planteamiento fundamental-ontológico de Heidegger y el filosófico-antropológico de Scheler no atañe a los resultados que unos y otros hubieran sacado de sus respectivos estudios de la existencia humana, sino esencialmente a la cuestión de la fundamentación de la filosofia. La voluntad de empezar la filosofía no con el ser humano sino con el ser y de pensar al ser humano desde su relación con el ser hizo difícil la aproximación de Heidegger y de la Antropología Filosófica e hizo también que durante décadas se utilizó con muchísima cautela la postura heideggeriana en los debates antropológicos (el filósofo Hans Blumenberg llega a hablar de una "prohibición antropológica" (Anthropologieverbot) en la fenomenología tanto heideggeriana como husserliana (Blumenberg, 2006, p. 60). Esta prohibición se va superando paulatinamente en la filosofia actual, legitimándose por el descubrimiento de algunos textos heideggerianos en los que él mismo se dejó llevar por reflexiones de alcance antropológico, sin obviamente abandonar el planteamiento de la analítica existencial. Dos textos son importantes en este sentido:

1) En el curso del invierno 1928/29, Einleitung in die Philosophie (Heidegger, 1996), Heidegger se apoya en las investigaciones de Ernst Cassirer, principalmente en Das mythische Denken (1925). Heidegger publicó en 1928 una reseña del libro de Cassirer en el Deutsche Literaturzeitung (Heidegger, 1977, p. 255-270), para elaborar una reflexión acerca del origen mítico de la existencia humana y de su descubrimiento de las preguntas filosóficas (\$§ 41-44). El ensayo de Heidegger anuncia curiosamente lo que filósofos como Hans Blumenberg o Peter Sloterdijk intentarán hacer años después: mostrar el origen de la fenomenología y de la filosofia a partir de consideraciones de naturaleza antropológica.

2) Encontramos también, en el curso del invierno de 1929/30, Die Grundbegriffe der Metaphysik. Welt, Endlichkeit, Einsamkeit (Heidegger, 1983), un intento bien conocido de pensar la diferencia entre la piedra, el animal y el ser humano que, a pesar de sus matices esencialmente ontológicos, recuerda los ensayos de Max Scheler o de Helmuth Plessner acerca de los grados que existen dentro de lo vivo. Aquí también Heidegger se dejó tentar por una utilización de su ontología fundamental en el contexto antropológico que le es en principio ajeno. 
Pero para entender mejor qué sentido tiene la "prohibición antropológica" en Heidegger, es preciso volver a su primera formulación en el $\S 5$ de Ser y tiempo. Según escribe ahí Heidegger, la analítica del Dasein que elabora en la obra no tiene otra tarea que "la elaboración de la pregunta por el ser" y, por tanto, el contenido de dicha analítica no puede ser leído como si contribuyese a un proyecto antropológico. Sin embargo y como lo subraya Heidegger mismo, sí permite que en el futuro se elabore sobre las bases del análisis existencial algo como una antropología filosófica:

La analítica del Dasein así concebida está orientada por entero hacia la tarea de la elaboración de la pregunta por el ser, que le sirve de guía. Con esto se determinan también sus límites. Ella no puede pretender entregarnos una ontología completa del Dasein, como la que sin duda debiera elaborarse si se quisiera algo así como una antropología 'filosófica' apoyada sobre bases filosóficamente suficientes. En la perspectiva de una posible antropología o de su fundamentación ontológica, la siguiente interpretación proporciona tan solo algunos 'fragmentos', que no son, sin embargo, inesenciales (Heidegger, 1927, p. 17).

Así, la prohibición antropológica tiene que ver fundamentalmente con 1) la interpretación de la analítica del Dasein como un ensayo de antropología filosófica o 2) la interpretación de la antropología filosófica (la de Scheler, por ejemplo) como una analítica del Dasein. Pero nunca prohíbe Heidegger que se utilice la analítica del Dasein como fundamentación "filosóficamente suficiente" de una posible y futura antropología filosófica. En esta dirección se orienta nuestra consideración antropológica acerca de los fenómenos de la enfermedad y de la salud. Pero antes de mostrar qué tipo de contribución la analítica existencial puede aportar a este cuestionamiento, es importante aclarar el significado que pueden tener enfermedad y salud en los debates actuales.

\section{SALUD Y ENFERMEDAD EN SENTIDO TEÓRICO-MÉDICO}

Pasando revista a la literatura en torno a este tema, encontramos algunas definiciones de los conceptos de salud y de enfermedad. La Organización Mundial de la Salud (OMS), por ejemplo, define la salud como "un estado de completo bienestar físico, mental y social, y no solamente la ausencia de afecciones o enfermedades" (Preamble, 1946). En dicha definición queda manifiesto que la salud es comprendida como un estado que va más allá de la mera ausencia de una enfermedad. En efecto, la definición de la OMS resulta tan amplia que ella representa más bien un ideal de lo que debería entenderse por salud. Pues en la mayoría de los casos muy pocas personas (o sus médicos) afirmarían tener un "estado de completo bienestar físico, mental y social". Por ello, la definición de la OMS ha sido considerada como un "concepto límite" (Grenzbegriff) (Hauser, 2011, p. 176).

Paralelamente es posible identificar una amplia gama de diversas definiciones de salud. En un estudio reciente, Schmidt enumera y analiza diez definiciones distintas de salud. De acuerdo con él, la salud puede ser entendida en relación con distintas perspectivas: como "armonía", como "lucha", como "momento de un 
proceso dialéctico", como "jerarquía", como "potencialidad" o como "mecanismo de efecto causal", entre otras interpretaciones (Schmidt, 2010). Del mismo modo en que existen diversas definiciones de salud, también contamos con diversas teorías de la salud. No obstante, la mayoría de ellas pertenecen (en mayor o menor grado) o bien a la posición normativista que propone una teoría holística de la salud o bien a la posición naturalista apoyada en una teoría anatómica-biológica de la salud. El defensor más famoso del enfoque naturalista es Christopher Boorse (1997).

Boorse desarrolla su Teoría Bioestadística de la Salud (Bio-Statistical Theory) en la que define la enfermedad como una falla objetiva de la función normal de un organismo o ser vivo. Así, Boorse sostiene la tesis de que enfermedad y salud constituyen conceptos neutros, empíricos y objetivos, en cuanto estos se basen en funciones o disfunciones biológicas naturales. De este modo, la posición naturalista de Boorse (1997) considera que "enfermedad", es un concepto puramente descriptivo al que escapan todo tipo de valoraciones subjetivas: The fundamental idea is that a pathological conditionis a state of statistically species subnormal biologicalpartfunction, relative to sex and age (p. 684). La enfermedad o patología se define, de acuerdo con Boorse, tomando como criterio de salud el funcionamiento "normal" del hombre en tanto partícipe de la especie homo sapiens. Para Boorse, la salud de un organismo está fundada en el orden natural de un organismo, siendo esto aquello que corresponde a su estado biológico normal, a su fisiología: Very important is that the biostatistical theory (BST) is, and always has been, a dynamic account of normal physiology (p. 685). Un organismo que vive de acuerdo con su eficiencia normal típica, debe ser capaz, siguiendo a Boorse, de alcanzar satisfactoriamente dos metas fundamentales: supervivencia y reproducción. La noción de normalidad estadística se ubica por tanto en el centro de su teoría de salud, ya que solo tomando en cuenta esto es posible determinar si un individuo está sano o enfermo. Según Boorse, un organismo puede estar enfermo en sentido teórico objetivo (a lo que Boorse llama disease) como también en sentido subjetivo (para ello utiliza la palabra illness).

Un ser vivo es normal y está sano si, por consiguiente, sigue la estructura funcional biológica "normal" y típica de su especie. Considerando lo anterior, se evidencia que Boorse comprende el concepto de salud de forma negativa: pues se trata de un concepto que es definido desde la ausencia de la enfermedad, en tanto la enfermedad es constatada como un obstáculo o impedimento del funcionamiento típico y normal del organismo (p. 4). En este sentido, Boorse define la salud de esta forma: Health in a member of the reference class is normal functionalability: thereadiness of each internal part to perform all its normal functions on typical occasions with at least typical efficiency (Boorse, 1977, p. 562).

La segunda teoría de la salud corresponde a la posición de los normativistas. A este enfoque pertenecen la mayoría de los teóricos de la salud y la mayoría de ellos tienen en común que consideran la salud y la enfermedad como conceptos normativos. Contrario a la posición de Boorse, los normativistas argumentan que el 
diagnóstico médico no consiste en la descripción de datos empíricos, sino que dichas descripciones siempre contienen valoraciones subjetivas, culturales o éticas. De acuerdo con los normativistas, para determinar el estado de salud de una persona no es suficiente dar con datos cuantitativos o estadísticos, sino que esto requiere de un diagnóstico holístico, una evaluation of the general state ofthe person (Nordenfelt, 2007, p. 6). Uno de los enfoques más discutidos de los normativistas corresponde la teoría de Lennart Nordenfelt, llamada Holistic Theory of Health (HTH), (Nordenfelt, 2007). Las teorías de Boorse y de Nordenfelt representan las teorías de la salud más influyentes en la actualidad.

De acuerdo con la teoría de la salud de Nordenfelt (2001), la salud no se identifica con la ausencia de la enfermedad, sino que ella refiere a la "capacidad" (ability) de alcanzar "metas vitales" (vital goals): Disabilityis a negative notion presupposing the semantic content of its positive contrary, ability. This gives the analysis of ability a primary place in my theory of health (p. 67, nota 2). En su teoría de la salud Nordenfelt distingue entre varios conceptos: enfermedad, sufrimiento, dolor e impedimento y aboga por la centralidad del concepto de impedimento.

De acuerdo con esta definición, una persona debe ser considerada saludable si cuenta con determinadas capacidades que le permiten alcanzar una meta vital determinada (no arbitraria). En este sentido, Nordenfelt define a habilidad como una característica que debe ser evaluada en relación con tres aspectos: al agente en cuestión, a su meta (o metas) en cuestión y a las circunstancias en las que aquel agente actúa. Nordenfelt ciertamente no establece una lista concreta de aquello que constituyen "metas vitales", sino que él ofrece tres requisitos formales para así determinar subjetivamente su contenido. Nordenfelt (2007) define las metas vitales en relación con un bienestar o felicidad a largo plazo: $A$ is completely healthy if, and only if, A has the ability, given standard circumstances, to reach all his or her vital goals (p. 6). De este modo, la salud es interpretada como un requisito fundamental para tener una vida satisfactoria, es decir, para alcanzar una buena vida. En relación con lo anterior, una enfermedad no implica, de acuerdo con Nordenfelt, un defecto anatómico del cuerpo "normal" como en el caso de la teoría de Boorse, sino una situación percibida como problemática: My basic idea is that the notion of illness when it comes to humans has its basis in the existence of a perceived problem (p. 6) El reconocimiento subjetivo de una situación o estado como problemático constituye pues, el requisito fundamental, siguiendo a Nordenfelt, para buscar ayuda médica.

\section{LA "ENFERMEDAD EXISTENCIAL"}

Como hemos mencionado antes, Heidegger evoca en el análisis de la existencia que lleva a cabo en Ser y tiempo la posibilidad de concebir la enfermedad como un "fenómeno existencial". ¿En qué consiste concretamente un fenómeno existencial? Este puede ser resumido de la forma siguiente: un fenómeno existencial -existenzial y no existenziell - es un fenómeno que posee, para decirlo en términos kantianos, un "estatuto 
trascendental" y que tiene que ver con las condiciones de posibilidad de los fenómenos "existentivos", existenziell. Por tanto, concebir la enfermedad como un fenómeno existencial la convertiría no tanto en un acontecimiento que encontramos de manera óntica en la existencia humana, sino más bien en algo que determina la existencia humana a ser de tal $u$ otra forma.

La muerte es un ejemplo claro de fenómeno existencial. La muerte no es un fenómeno que vendría al encuentro en la existencia humana en un momento u otro, sino más bien algo que determina por completo nuestra relación con el tiempo y que, por tanto, determina cada una de las facetas de nuestra existencia. No pasa lo mismo con los intercambios económicos, los ritos ligados a la ingesta de alimentos o los usos que podamos hacer del mundo animal y vegetal. Son fenómenos existentivos importantes, sin lugar a dudas, pero no son determinantes en un nivel existencial como lo son, por ejemplo, fenómenos destacados por Heidegger como el mundo, el habla o el otro.

Como sabemos, en los textos de Heidegger de 1920, la muerte desempeña un papel importante. El trato que da Heidegger al tema no tiene ninguna connotación tétrica y no pretende, en ningún caso, ser una justificación para el suicidio (Vetter, 2014, p. 352). Ahí, la muerte no se piensa como un hecho biológico, sino a partir del rasgo propio de la existencia humana que es el "adelantarse a la muerte". Por muy sano y vivo que uno sea, su existencia siempre es, explícita o implícitamente, un camino hacia la muerte que se resume en la fórmula sum moribundus (Heidegger, 1979, p. 437-438). Querámoslo o no, somos todos "moribundos", es decir, que desde el día de nuestro nacimiento, hemos empezado a morir.

Por tanto, el tratamiento que reserva Heidegger en su análisis existencial al fenómeno de la muerte no es de ninguna manera biológico. No considera al Dasein como vida en general sino como existencia (o a lo sumo, como vida humana) y la muerte se ve como un problema ontológico ligado a nuestra comprensión de la temporalidad. Pero a pesar de estar muriendo en su "estar vuelto hacia la muerte" (Sein zum Tode), el Dasein también tendrá que acabar su vida biológica. A este acabar, Heidegger le da dos nombres, dependiendo de si se trata del acabar de la existencia humana o el acabar de la vida animal y vegetal: habla de 1) "dejar de vivir" (Ableben) en el caso de los seres humanos y de 2) "fenecer" (Verenden) en el caso de los animales y de las plantas (Heidegger, 1927, p. 247; Heidegger, 1983, p. 388). Esta distinción tiene una razón evidente: la muerte biológica no le llega al hombre como algo imprevisto, sino como algo esperado y a menudo temido. A las plantas y a los animales, sin embargo, el fin les llega sin que puedan anticiparlo.

En el tratamiento que Heidegger hace del fenómeno de la muerte, nos interesa sobre todo la distinción que se establece entre la manera animal de morir y la manera propia de la existencia humana, donde el "dejar de vivir" del ser humano tiene primacía filosófica frente al "fenecer" meramente biológico. Pero no deja de reconocer que existen dos maneras de abordar un acontecimiento único. La cuestión que nos interesa y 
que Heidegger plantea sin responder es si podríamos establecer la misma distinción al nivel del fenómeno de la enfermedad. Podemos suponer-veremos por qué enseguidaque la vivencia de la enfermedad por parte de los animales y de los hombres es inconmensurable. Por tanto, habría una especificidad del enfermar humano que es posible estudiar. Pero preguntamos: ¿también cabe la posibilidad de estudiar el enfermar no del ser humano sino del Dasein, de la existencia? ¿Enferma la existencia, el Dasein, o solamente el cuerpo, es decir, el espacio en el que se "extiende" el Dasein? ¿Tiene sentido estudiar la enfermedad como fenómeno existencial más allá del mero acontecimiento biológico propio de la vida humana?

Para empezar, es importante insistir en la distinción que existe entre la vivencia animal y humana de la enfermedad, algo que no podemos dar por supuesto. Esta distinción solamente se puede hacer desde una interpretación particular de lo que es la enfermedad. Siguiendo la definición naturalista de la enfermedad de Boorse, no tendría sentido establecer una tal distinción. Pues según la teoría bioestadística, la enfermedad es entendida como una anomalía objetiva (estadísticamente descriptible) de la función normal de un organismo y en relación con su especie. Esta definición no permite entender la enfermedad como algo fundamentalmente distinta en el hombre y en el animal. Para que se pueda realmente distinguir entre el enfermar humano y el enfermar animal, es necesario adoptar otra postura frente a la enfermedad, en concreto la postura normativista, ya que en esta teoría la enfermedad se entiende como un fenómeno que implica una disminución de las capacidades del enfermo para alcanzar sus metas vitales. En este sentido, una persona enferma es una persona que no puede hacer lo que sí podría estando bien de salud. Se experimenta la enfermedad no como una anomalía respecto de una norma sino más bien como una limitación en nuestras capacidades, es decir, de lo que uno puede hacer.

Esta definición de la enfermedad es la que permite pensar la enfermedad como un fenómeno existencial en la medida en que implica una comprensión de la experiencia del enfermar que la convierte en algo propio del ser humano. Esta experiencia de la disminución de las posibilidades solamente es accesible a un ente que se proyecta hacia posibilidades y que se entiende a sí mismo no tanto a partir de su estado presente como de su poder-ser. En Ser y tiempo Heidegger emplea el fenómeno de la muerte como base de su reflexión acerca del ser posible del Dasein, pero solamente aborda de pasada el de la enfermedad. Solo será mucho más tarde, en los seminarios que impartirá en Zollikon en 1960, cuando Heidegger se interese de cerca al fenómeno de la enfermedad que define entonces como "una pérdida de libertad, una limitación de la posibilidad de vida" (Heidegger, 1987, p. 202), en consonancia, si queremos, con la definición normativista que mencionamos antes.

En Ser y tiempo, Heidegger analiza el ser para la muerte de la existencia humana como - según la fórmula clásica- "la posibilidad de la imposibilidad" (Heidegger, 1927, p. 262). Frente a este ser para la muerte, la enfermedad permitiría experimentar algo distinto: la disminución o el aumento de mis posibilidades, es decir, 
una variación de grado en lo que concierne a las posibilidades hacia las que proyecto mi propio ser. Si entendemos la esencia de la existencia humana como poder-ser, es decir, como la capacidad de ser esto o lo otro, la enfermedad pone de manifiesto la posibilidad de una variación en el ámbito de lo posible. Algunas enfermedades (no hablamos de la gripe, sino de enfermedades crónicas o quizás de forma más evidente de la enfermedad mental), la capacidad propia se ve radicalmente truncada.

Podemos aquí intentar pensar retrospectivamente esta definición del último Heidegger de la enfermedad como limitación de la posibilidad de vida-interpretada también como pérdida de libertad - dentro del marco del análisis existencial, de tal forma que la enfermedad pueda cobrar la importancia de un fenómeno existencial. Si entendemos libertad y posibilidad desde una perspectiva óntica, es decir, como la capacidad de elegir entre llevar a cabo un acto u otro sin que mi voluntad esté determinada por fuerzas ajenas, entonces el fenómeno de la pérdida o de la limitación de esta capacidad no aporta luz al problema existencial como tal. Pero si nos acercamos al sentido que Heidegger otorga a la posibilidad y a la libertad en los años que siguen inmediatamente la publicación de Ser y tiempo, en los cursos y las publicaciones de fines de 1920, la limitación de las posibilidades y la pérdida de libertad se convierten en fenómenos que no atañen únicamente a molestias de la vida corriente, sino en algo que toca la estructura ontológica de la existencia humana. En el contexto kantiano de fines de 1920 cuando Heidegger se vuelca completamente en una interpretación de la Crítica de la razón pura, la finitud aparece de repente como el tema central del pensamiento heideggeriano. Es cierto que ya aparece el tema en Ser y tiempo, cuando se evoca la finitud del tiempo de la existencia humana que la muerte pone de manifiesto. En los textos siguientes, sin embargo, la finitud se presenta por una parte como el tema kantiano de la finitud del conocimiento (entregado a la intuición empírica, intuitus derivativus) y, por otra, como algo encontrado, según la formulación curiosa de Kant und das Problem der Metaphysik, “dentro del ser humano" (Heidegger, 1977, p. 229).

En razón de lo anterior, la finitud es pensada no como el descubrimiento de incapacidades propiamente ónticas (como nuestra incapacidad a volar, a pasar a través de las paredes o a dejar de alimentarnos indefinidamente), sino desde la experiencia del mundo como algo impuesto, algo que no hemos elegido y que choca contra nuestra libertad entendida como proyecto. Es esta tensión propia de la existencia humana entre posibilidad e imposición que Heidegger caracteriza en estos años como "proyecto arrojado" (geworfener Entwurf). Heidegger explica entonces la posibilidad de la relación con los entes que descubrimos en el mundo como el encuentro entre la proyección libre de un mundo y de la absorción restrictiva en el ente.

La limitación de las posibilidades de uno se entenderá, pues, como una pérdida de libertad en la medida en que el equilibrio entre "proyecto" (Entwurf) y "estararrojado" (Geworfenheit) se desajusta de tal forma que la parte que atañe a nuestro poder sobre el mundo disminuye en proporción a la parte que atañe a nuestra 
impotencia en el mundo. Independientemente de las consecuencias que pueda tener este desajuste, la experiencia de estas variaciones se podría interpretar como el descubrimiento de la finitud ontológica del Dasein que siempre llega al ente desde la comprensión que tiene del ser y que constituye para Heidegger el verdadero "sello" de la finitud de la existencia humana.

Esta lastra propia de la existencia humana es lo que la hace única frente a los animales y a los dioses: los primeros no pueden nunca abordar el ente en cuanto ente y los segundos, se supone, no necesitan de este rodeo hermenéutico. Si dejamos el problema de los dioses de lado, vemos -y es ciertamente el sentido que tiene la tesis acerca de la pobreza en el mundo propia de los animales- que el animal no puede de ninguna forma experimentar lo que Heidegger llama la pérdida de libertad o la limitación de las posibilidades de vida. El animal se mantiene absorbido en el ente, de tal forma que en ningún momento de su vida puede tomar sus distancias con la realidad y experimentar la muerte o la enfermedad (Heidegger, 1983, p. 388). Así, la vivencia de la enfermedad entendida como limitación de las posibilidades de vida pone de manifiesto un fenómeno existencial propio del único ser vivo que existe como tal: el Dasein. La enfermedad constituye, por tanto, un fenómeno destacado - existencial y no existentivo- que permite el descubrimiento por parte del Dasein de la finitud de su poder-ser.

\section{CONCLUSIÓN: SALUD Y PODER-SER}

A partir del análisis presentado se desprenden varias conclusiones. En primer lugar, hemos mostrado que siguiendo las descripciones de Heidegger acerca de la muerte, así como considerando retrospectivamente su comprensión de la enfermedad como una "pérdida de libertad" es posible proponer un planteamiento heideggeriano respecto de la enfermedad como fenómeno existencial. En este sentido, encontramos una cercanía estructural entre los conceptos de enfermedad y de muerte. Pues aquello que hace a la muerte un fenómeno existencial fundamental para el Dasein radica en Ser y tiempo justamente en su rol desvelador de la temporalidad auténtica y asimismo, de su ser como posibilidad. La muerte en tanto posibilidad constante permite una toma de distancia para con el vivir cotidiano del Dasein -y que normalmente es ignorante de la estructura ontológica y dinámica de la existencia-, haciendo posible una especie de retornar a sí mismo. De forma similar a la meditiatio mortis heideggeriana, también la experiencia de ciertas enfermedades pueden revestir una función individualizadora (qua principium individuationis) en la que el Dasein puede retornar a sí desde su estado impropio y reconocerse en su ser como ser posible. Desde antaño, el estado enfermo es entendido como una situación de crisis que es capaz de obstaculizar nuestro vivir cotidiano - especialmente el caso de enfermedades crónicas-. Ahora bien, dependiendo del tipo de enfermedad que nos atañe, podemos experimentar la enfermedad como situación de crisis existencial. Esta situación ya la reconoció Nietzsche con claridad, cuando sostiene que la enfermedad permite quizás 
no optimizarnos, pero sí "profundizarnos" (vertiefen) (Nietzsche, 2000, p. 350). En la medida en que experimentemos la enfermedad no desde el dolor empírico y positivo, sino más bien como una interrupción precisamente de la libertad que ejercemos de facto e irreflexivamente en nuestras vidas, entonces la enfermedad puede ocasionar una angustia existencial similar a la angustia ante la muerte. Mediante dicha forma de crisis ocurre una toma de distancia, un revalorar la libertad de acción que tenemos en la sanidad, pero sin ser genuinamente conscientes de ello.

En concordancia con el planteamiento heideggeriano de la enfermedad existencial que hemos presentado hasta ahora, la salud no es descubierta como un estado de ausencia o carencia de enfermedad como sostiene Boorse o como el estado "oculto" (verborgen) del que habla Gadamer (1993). Muy por el contrario, a diferencia de estos conceptos negativos (carencia, ausencia, etcétera), la salud sería entonces descubierta en un sentido absolutamente positivo, como condición de posibilidad para la acción. Si la enfermedad es considerada una "pérdida de libertad", la salud, en consecuencia, tiene que ser vista como un conservar, y así, posibilitar dicha libertad. Esta perspectiva holística de la salud, en la línea de las teorías normativistas de la salud, tiene importantes ventajas para la reflexión ético-teórica propias de la medicina actual: en cuanto la salud es entendida como una estructura compleja y que refleja la manera de ejercer libertad y soberanía, ella permite que la salud no sea reducida al mero bienestar del cuerpo anatómico-biológico. En este sentido, aquello considerado como el cuerpo "normal", capaz de desplegar ciertas funciones con determinadas capacidades "promedio" (por ejemplo en función de la reproducción y la supervivencia), pierde su peso normativo. En tanto la salud no es identificada con un determinado estado físicopsíquico, localizable, cuantificable y explicable mediante conceptos naturalistaspositivos, sino más bien con una condición de posibilidad más amplia y que involucra mucho más que el mero llevar a cabo una vida "normal", desarrollando funciones típicas de la especie homo sapiens. Así, entender la salud como posibilidad de libertad significa a fin de cuentas permitir el desarrollo individual y autónomo de proyectos vitales, proyectos que bien pueden ir acorde al entendimiento médico generalizado y así restringido de salud, pero que también puede ser independiente de este. Una enfermedad que me impide, por ejemplo, desplazarme con rapidez de un lado a otro puede motivar una reflexión acerca del rol que tiene la movilidad en general en nuestras vidas; replantearse lo importante que es el deporte o la velocidad, lo dependiente o independiente que somos de un medio de transporte determinado o el valor que puede tener la caminata diaria por el vecindario. La enfermedad puede ser ocasión de replantearse el valor de estados, acciones o costumbres que normalmente e irreflexivamente aceptamos y desde cuya comprensión nos desenvolvemos sin cuestionarnos sus fundamentos. Por lo demás, esta concepción amplia de salud permite que un proyecto de vida distinto, minoritario y contrario al modelo naturalistareduccionista de salud (por ejemplo en algunos casos de intersexualidad) pueda ser comprendido como igualmente aceptable que otras formas de existencia con otros tipos 
de constitución biológica-anatómica. Suma sumarum: ser distinto es posible donde no se patologiza y normaliza lo distinto.

Sin embargo, pese al enorme potencial que tiene la visión heideggeriana de la salud y la enfermedad como hemos presentado hasta aquí, ella también trae consigo algunos desafios. Pues si bien la consideración existencial de la enfermedad trae ventajas éticas por ejemplo en relación con la apertura y tolerancia que esta visión implica para con proyectos vitales posiblemente distintos del estándar anatómico "normal" de nuestra especie, al mismo tiempo plantea problemas para distinguir situaciones efectivamente médicas de otras que no lo son. Pues si la enfermedad no es definida en términos naturalistas-positivos como postulan los normativistas, concordante justamente con la posición heideggeriana, ¿cómo distinguir entre experiencias que representan crisis existenciales no médicas (la pérdida afectiva, por ejemplo) de una enfermedad concreta? O mejor aún, ¿es suficiente caracterizar la enfermedad como una "pérdida de libertad" siendo que ciertamente existen situaciones y experiencias no médicas que coartan la libertad de forma radical como, por ejemplo, la educación religiosa adquirida por los padres o por la sociedad?

Frente a dicha crítica, debe hacerse hincapié en al menos dos aspectos. En primer lugar, que la enfermedad sea una pérdida de libertad no significa que la salud corresponda a un estado de pura libertad. Aquello sería incompatible con la comprensión heideggeriana de la facticidad del Dasein como un proyecto arrojado, es decir, como quien tiene libertad de hacerse, mas solo dentro de ciertos márgenes. La salud, pues, no está siendo considerada como un estado idealizado de pura autocreación. En segundo lugar, una manera que quizás nos permitiría dar respuesta al problema que efectivamente se presenta al quitar el fundamento naturalista para la consideración de la salud/enfermedad sería recalcar que la enfermedad al igual que la muerte no constituyen meras limitaciones de la libertad, sino en realidad, limitaciones en sentido estricto. Ambas experiencias permiten experimentar (si bien de modos distintos: la muerte mentada; la enfermedad percibida) la propia finitud de un modo particular, a saber, absolutamente ligada a la ipseidad. La enfermedad y la muerte me agobian y me atañen de un modo muy distinto a cómo me limita el saber solamente ciertos idiomas, seguir cierta religión u orientarme bajo ciertos valores; pues, mientras que lo último limita mi acceso al mundo, mas puede de algún modo ser superado o compensado, la limitación que nos atañe en el caso de la enfermedad o la muerte no son sorteables con la mera voluntad, suerte o medios materiales. La enfermedad me permite experimentar a nivel óntico aquella limitación fundamental y de tipo ontológico que constituye nuestros ser, pues ella retrotrae e individualiza a cada uno de nosotros en tanto seres finitos. En la medida de que experimentamos una determinada discapacidad o una enfermedad que no nos imposibilite la reflexión (es decir, una enfermedad libre de dolores agudos), dicha situación nos obliga a plantearnos lo que se ha sido, comparándolo con lo que se es y con lo que se esperaba ser. La enfermedad no solo provoca compararse con el funcionamiento "normal" físico estadístico de nuestra 
especie y así con otros (también puede ser comportamiento psíquico, mas ahí hay más dificultades para el diagnóstico médico), sino que de manera más originaria nos comparamos con nosotros mismos, con lo sido, lo siendo y lo por ser. A raíz de lo anterior, la consideración de la salud/enfermedad heideggeriana, como la hemos planteado aquí pese a posibles problemas, pareciera darnos indicios originales y hasta ahora no tematizados de este modo en la teoría, ética y filosofía de la medicina actual.

Este estudio es resultado del Proyecto FONDECYT DE INICIACIÓN 2015 $\mathrm{N}^{\circ} 11150298$ por 3 años, titulado: "El "paciente sano": Perspectivas éticas y filosóficas en tiempos de una medicina cada vez más tecnificada".

\section{OBRAS CITADAS}

Blumenberg, Hans (2006). Beschreibung des Menschen, Frankfurt am Main: Suhrkamp, 2006. trad. esp. G. Mársico, Descripción del ser humano, Buenos Aires: FCE, 2010.

Boorse, Christopher (1997). “A Rebuttal on Health", en J. M. Humber y R. F. Almeder (eds.), What is Disease? Totowa: Humana Press.

(1977). "Health as a Theoretical Concept", en Philosophy of Science, /44 (4): 542-573.

Cassirer, Ernst (1925). Philosophie der symbolischen Formen, Bd. 2: Das mythische Denken, Berlin.

Fischer, Joachim (2009). Philosophische Anthropologie. Eine Denkrichtung des 20. Jahrhunderts, Freiburg im Breisgau: Alber.

Gadamer, Hans-Georg (1993). Über die Verborgenheit der Gesundheit, Frankfurt am Main: Suhrkamp.

Gehlen, Arnold (1983). "Zur Geschichte der Anthropologie", en Philosophische Anthropologie und Handlungslehre, Gesamtausgabe, volumen 4, ed. Karl Siegbert Rehberg. Frankfurt am Main: Klostermann.

Hauser, Linus (2011). "Gesundheit und Krankheit im Kontext einer philosophischtheologischen Anthropologie”, en: P. Hensen y C. Kölzer, Die gesunde Gesellschaft. Sozioökonomische Perspektiven und sozialethische Herausforderungen. Wiesbaden: Springer.

Heidegger, Martin (1996). Einleitung in die Philosophie, GA 27, ed. O Saame y I. Saame-Speidel; trad. esp. Manuel Jiménez Redondo, Introducción a la filosofia. Valencia: Cátedra, 2001.

_ (1987). Zollikoner Seminare, Frankfurt am Main, Klostermann; trad. esp. Ángel Xolocotzi Yáñez, Seminarios de Zollikon. Barcelona: Herder, 2013.

_ (1983). Die Grundbegriffe der Metaphysik. Welt-Endlichkeit-Einsamkeit, Gesamtausgabe (en adelante GA), tomo 29/30, ed. F. W. von Herrmann, 
Frankfurt am Main: Klostermann, trad. esp. Alberto Ciria, Los conceptos fundamentales de la metafisica. Mundo, Finitud, Soledad. Madrid: Alianza. 2007.

(1979). Prolegomena zur Geschichte des Zeitbegriffs, GA 20, Frankfurt am Main: Klostermann; trad. esp. Jaime Aspiunza, Prolegómenos para una historia del concepto de tiempo. Madrid: Alianza, 2006.

_ (1978). Metaphysische Anfangsgründe der Logik im Ausgang von Leibniz, GA 26, ed. K. Held, Frankfurt am Main, Klostermann; trad. esp. J. J. García Norro, Principios metafisicos de la lógica. Madrid: Síntesis, 2009.

— (1976). Briefüber den "Humanismus", en Wegmarken, GA 9, ed. F. W. von Herrmann. Frankfurt am Main: Klostermann; trad. esp. H. Cortés y A. Leyte, Hitos. Madrid: Alianza, 2000.

(1977). Kant und das Problem der Metaphysik, GA 3, ed. F. W. von Herrmann, Frankfurt am Main: Klostermann, 1954: 255-270; trad. esp. Gred Ibscher Roth. México: FCE.

_ (1927). Sein und Zeit, Halle an der Saale, Niemeyer; trad. esp. J. E. Rivera, Ser y tiempo. Santiago de Chile: Universitaria, 1997/Madrid: Trotta, 2003.

Nietzsche, Friedrich (2000). "Die fröhliche Wissenschaft", en: Kritische Studienausgabe (KSA), tomo 3, ed. G. Colli y M. Montinari, dtv.

Nordenfelt, Lennart (2007). "The Concepts of Health and Illness Revisited", en Medicine, Health Care and Philosophy 10.

Nordenfelt, Lennart et al. (2001). Health, Science and Ordinary Language. Amsterdam: Rodopi.

Preamble to the Constitution of the World Health Organization as adopted by the International Health Conference. (1946). New York. Versión en español: http://apps.who.int/gb/DGNP/pdf_files/constitucion-sp.pdf Rescatado el 23/5/2016.

Scheler, Max (1987). Schriften aus dem Nachlaß. En Gesammelte Werken (GW), tomo XII: Philosophische Anthropologie, ed. M. S. Frings.

— (1928). Die Stellung des Menschen im Kosmos. Darmstadt.

_ (1926). Mensch und Geschichte, en Späte Schriften, Gesammelte Werken, tomo IX, ed. M. S. Frings. Bonn: Bouvier Verlag, 1979; trad. esp. J. J. Olivera, La idea del hombre y la historia. Buenos Aires: La Pléyade, 1972.

Schmidt, Josef M (2010). "Gesundheit! -Geschichte und Konzepte des Leitbegriffs der Medizin", en Wiener klinische Wochenschrift, 122: 538-542.

Vetter, Helmuth (2014). Grundriss Heidegger. Ein Handbuch zum Leben und Werk, Hamburg: Felix Meiner. 